\title{
MÉTODOS ALTERNATIVOS PARA ESTABILIZAÇÃO DE FRATURAS DE RÁDIO E ULNA EM CÃES E GẢTOS
}

\author{
ALTERNATIVES METHODS FOR FRACTURE STABILIZATION OF RADIO AND ULNA IN DOGS \\ AND CATS
}

\author{
Amaro Fábio de Albuquerque Souza ${ }^{1}$ Eduardo Alberto Tudury ${ }^{2}$ Ana Maria Martins da Silva ${ }^{3}$ \\ Ilvio Mendes Vidal ${ }^{4}$
}

RESUMO

Devido à grande casuística de fraturas diafisárias $e$ metafisárias distais de rádio e ulna em pequenos animais e ao alto custo dos implantes ortopédicos recomendados na literatura, tornou-se necessário o estudo de novos métodos de estabilização, que diminuíssem os inconvenientes inerentes à técnica de estabilização radial mediante à colocação de um único pino intramedular, sem encarecer o procedimento. Para tais fins, foram testados em 17 animais (15 caninos e 2 felinos), a imobilização interna do rádio com um pino intramedular, complementado por uma dupla hemicerclagem interfragmentar em X (10 animais), e a colocação de um pino intramedular no rádio e outro na ulna (7 animais), visando com isto, anular todas as forças atuantes no foco de fratura. Esses métodos, complementados com imobilização externa, demonstraram ser eficientes do ponto de vista cirúrgico, diminuindo significativamente os custos, o tempo anestésico, a freqüência de cuidados pós-operatórios, o tamanho da incisão e o grau de exposição óssea quando comparados com outros métodos citados na literatura.

Palavras-chave: fixação de fraturas, rádio e ulna, cirurgia de cães e gatos.

\section{SUMMARY}

Due to the great number of distal diaphyseal and metaphyseal radio and ulna fractures in small animals and high cost of orthopedical implants recommended in literature, the study of new methods of stabilization for reducing inherent inconveniences to such techniques and costs of orthopedical surgeries was necessary. For such objective, an internal immobilization method of the radio with a single intramedullary pin complemented by a double interfragmentary hemicerclage in
$X$ (10 animals) and the method of placement of an intramedullary pin in the radio and another in the ulna (7 animals), were tested in 17 animals (15 canine and 2 felines), thus trying, to block all forces present in the fracture focus. These methods, demonstrated to be surgically viable, diminishing costs, anesthesic period, frequency of aid after surgery, incision size and degree of bone exposition when compared to other methods mentioned in the revised literature.

Key words: fracture fixation, radio and ulna, dog and cat surgery.

\section{INTRODUÇÃO}

Em cães, o rádio não apresenta conformação uniforme, sendo a diáfise e a metáfise achatadas em sentido craniocaudal e ligeiramente curvas. Seu canal medular é normalmente uniforme em tamanho e mais largo no sentido médio-lateral que craniocaudal. O canal medular da ulna é mais largo na porção proximal, estreitando-se distalmente ao longo de seu trajeto, sendo que em cães de pequeno porte pode ser muito estreito ou não existir (NEWTON \& NUNAMAKER, 1985).

Enquanto MUIR (1997) afirma que as fraturas antebraquiais acometem $17 \%$ dos cães com fraturas, FOSSUM et al. (1997) dizem que as de rádio e ulna compreendem $8,5 \%$ a $18 \%$ das fraturas em cães e gatos. Todos os tipos de fraturas podem acometer tanto o rádio quanto a ulna ou ambos os

\footnotetext{
${ }^{1}$ Médico Veterinário Autônomo, Estagiário do Projeto de Extensão “Ortopedia e Neurocirurgia Animal”, Departamento de Medicina Veterinária, Universidade Federal Rural de Pernambuco (UFRPE).

${ }^{2}$ Médico Veterinário, Doutor, Professor Adjunto 1, Departamento de Medicina Veterinária, UFRPE, Rua Dom Manoel de Medeiros s/n, Dois Irmãos, 52171-900, Recife - PE. E-mail: tudury@nelore.npde.ufrpe.br Autor para correspondência.

${ }^{3}$ Médico Veterinário, Residente do Hospital Veterinário da UFRPE.

${ }^{4}$ Discente do curso de Medicina Veterinária, Estagiário do Projeto de Extensão "Ortopedia e Neurocirurgia Animal”. Departamento de Medicina Veterinária, UFRPE.
} 
ossos (ARCHIBALD, 1974; NEWTON \& NUNAMAKER, 1985; PIERMATTEI \& FLO, 1998). A causa mais comum de fraturas rádioulnares são atropelamentos (MUIR, 1997), sendo os animais jovens mais susceptíveis (FOSSUM et al., 1998). Em cães de raças pequenas, podem ocorrer após um mínimo trauma, como por exemplo um pulo ou queda (MUIR, 1997), afetando principalmente a região distal da diáfise radial (HERRON, 1974; WHITTICK, 1978; NEWTON \& NUNAMAKER, 1985; FOSSUM et al., 1997; MUIR, 1997). As fraturas rádio-ulnares são muito predispostas à não união óssea, união retardada ou à má união angular (DEANGELIS et al., 1973; ARCHIBALD, 1974; HERRON, 1974;WHITTICK, 1978; NEWTON \& NUNAMAKER, 1985; PIERMATTEI \& FLO, 1998). Basicamente, o problema parece decorrer do mau alinhamento das extremidades da fratura, devido ao pequeno diâmetro ósseo (HERRON, 1974), pobre suprimento vascular (DEANGELIS et al., 1973; HERRON, 1974; NEWTON \& NUNAMAKER, 1985), pouca musculatura de suporte e forte tensão exercida pelos músculos, flexor carpal e flexor digital, que deslocam os fragmentos caudal e lateralmente (HERRON, 1974).

Para reduzir as fraturas de rádio e ulna, a literatura revisada cita dois métodos, fechado ou conservador (muletas, moldes, gesso, talas e bandagens) e aberto ou cirúrgico (placas e parafusos ortopédicos, fios de cerclagem, pinos intramedulares e pinos percutâneos, mais fixadores externos) (NEWTON \& NUNAMAKER, 1985; FOSSUM et al., 1997; PIERMATTEI \& FLO, 1998). A decisão de realizar uma redução aberta ou fechada é feita com base na configuração da fratura. As vantagens e desvantagens de uma redução aberta ou fechada precisam ser medidas (FOSSUM et al., 1997). De acordo com MATTHIESEN (1983), a escolha do tipo de redução depende do tipo de fratura, peso, raça e idade do animal, acompanhamento do proprietário e fatores econômicos.

A filosofia básica, relacionada à reconstrução de ossos fraturados, baseia-se na redução anatômica ou na aproximação dos maiores fragmentos. Para que haja uma correta cicatrização óssea, os implantes ortopédicos devem anular todas as forças atuantes no foco de fratura, forças essas intituladas como rotação, angulação, compressão, deslocamentos (laterais e longitudinais) e distração dos fragmentos da fratura (SINIBALDI \& BOUDREAU, 1992). Devidamente aplicadas, as placas ortopédicas promovem a mais efetiva forma de fixação de fraturas que o cirurgião dispõe. Elas são efetivas na neutralização de todas as forças existentes no foco de fratura (SCHWARZ, 1991). Um pino intramedular somente é efetivo na neutralização de forças de angulação (DEANGELIS $\boldsymbol{e} \boldsymbol{t} \boldsymbol{a l}$., 1973; SCHWARZ, 1991) e essa habilidade é diretamente proporcional ao diâmetro do mesmo (SCHWARZ, 1991).

As placas e os parafusos ortopédicos demonstraram ser mais eficientes no tratamento das fraturas diafisárias de rádio e ulna (DEANGELIS $\boldsymbol{e} t$ al., 1973; NEWTON \& NUNAMAKER, 1985; FOSSUM et al., 1997; MUIR, 1997; PIERMATTEI \& FLO, 1998), porém, são mais caras que os pinos intramedulares e fios de cerclagem, necessitando, para sua aplicação, de maior número de instrumentos e maior exposição óssea, além de interferirem no suprimento sangüíneo cortical sob a placa e levarem ao enfraquecimento ósseo (PIERMATTEI \& FLO, 1998).

Os pinos intramedulares necessitam de menos equipamentos para sua aplicação, ocasionando menor exposição do osso, trauma nos tecidos adjacentes e prejuízo ao suprimento sangüíneo periosteal (FOSSUM et al., 1997; PIERMATTEI \& FLO, 1998). Para sua remoção não necessitam de uma extensa incisão, diminuindo, quando comparados com as placas ortopédicas, o tempo da cirurgia, da anestesia e os custos inerentes à mesma (PIERMATTEI \& FLO, 1998). Seu uso é limitado devido ao fato de não conterem a instabilidade rotacional e pela possibilidade de lesarem a articulação carpo-radial durante sua introdução e permanência (FOSSUM et al., 1997; BOJRAB et al., 1998; PIERMATTEI \& FLO, 1998).

Complicações como união retardada, não união óssea e doenças degenerativas das articulações do cotovelo e carpal são associadas às fraturas de rádio e ulna tratadas com pinos intramedulares (DEANGELIS et al., 1973; FOSSUM et al., 1997; BOJRAB et al., 1998). Esses problemas são referidos a $80 \%$ dos animais submetidos a essa técnica (LAPPIN et al., 1983). Embora alguns autores citem o sucesso do tratamento de fraturas de rádio e ulna com pinos intramedulares, até em cães de raças gigantes (KELBER \& CHARLEBOIS, 1958; HERRON, 1974; PIERMATTEI \& WIND, 1976), outros, (LAPPIN et al., 1983; NEWTON \& NUNAMAKER, 1985; FOSSUM et al., 1997; MUIR, 1997; BOJRAB et al., 1998; PIERMATTEI \& FLO, 1998) desaconselham o uso dessa técnica, já que, em decorrência da estabilidade oferecida não ser completa, pode levar à não união fibrosa, e pela possibilidade de que as pontas distais dos pinos possam lesar definitivamente a articulação radiocarpal. Ocasionalmente, tem-se utilizado um pino intramedular na ulna para estabilizar fraturas distais de 
rádio e ulna em cães de raças gigantes, complementando a imobilização por meio de muletas, talas ou placas ortopédicas no rádio (NEWTON \& NUNAMAKER, 1985).

O uso de fios de aço flexíveis (cerclagem), no tratamento de fraturas, tem conseguido grande aceitação em ortopedia veterinária, devido a características favoráveis como fácil aplicação, baixo custo em relação a outros implantes ortopédicos e versatilidade (BOJRAB et al., 1998). As cerclagens são utilizadas primariamente para neutralizar forças de cisalhamento (deslocamentos lateral e longitudinal), contudo também se mostram bastante efetivas para neutralizar forças de rotação, dependendo da forma de implantação (SINIBALDI \& BOUDREAU, 1992). Quando aplicadas de forma correta, não prejudicam o suprimento vascular nem predispõem a não união óssea (NEWTON \& HOHN, 1974; HINKO \& RHINELANDER, 1975; PIERMATTEI \& FLO, 1998). A forma de colocação dos fios de aço que promove mais estabilidade antirotacional é a dupla hemicerclagem interfragmentária cruzada (em X), sendo que, à medida que aumenta a distância entre os orifícios de passagem da cerclagem e a linha de fratura, diminui-se a capacidade anti-rotacional desta dupla hemicerclagem (BLASS et al., 1985).

Esta pesquisa teve como objetivos avaliar duas técnicas de imobilização de fraturas rádioulnares de cães e gatos: 1) um pino intramedular no rádio, complementado com uma dupla hemicerclagem interfragmentária em $\mathrm{X}$, mais imobilização externa com tala de PVC, e 2) um pino intramedular no rádio e outro na ulna, complementado com o mesmo tipo de tala, ambas pretendendo estabilização eficiente a baixo custo.

\section{MATERIAL E MÉTODOS}

Foram utilizados 17 animais (15 caninos e 2 felinos), pacientes do Hospital Veterinário da UFRPE. O grupo I (Tabela 1), composto por dez animais, foi submetido ao método de redução com pino intramedular suplementado com a dupla hemicerclagem interfragmentária em X (Figura 1 e 2) e o grupo II (Tabela 2), composto por sete animais, foi
Resenha, tipo de fratura, evolução e complicações dos cães nos quais utilizou-se a técnica de um pino intramedular no rádio e dupla hemicerclagem interfragmentária em X mais tala, para estabilização de fraturas de rádio e ulna. Recife, 1999.

Tipo de Evolução e complicações

fratura

FDRU ${ }^{1} \quad$ Cicatrização óssea normal, sem retirada$$
9 \mathrm{~m}, 4 \mathrm{~kg}
$$

dos implantes

Quebra do pino, não união óssea

$\mathrm{m}, 2,5 \mathrm{~kg}$

FDRU

FDRU

Cicatrização óssea normal, sem retirada dos implantes

FDRU Cicatrização óssea normal, sem retirada dos implantes

FDRU Cicatrização óssea normal, sem retirada dos implantes

FDRU Cicatrização óssea normal, retirada do pino do rádio

Cicatrização óssea normal, sem retirada dos implantes

Cicatrização óssea normal, sem retirada dos implantes

Cicatrização óssea normal, retirada do pino do rádio

Pino no espaço articular, nova cirurgia para melhorar o sepultamento do pino 


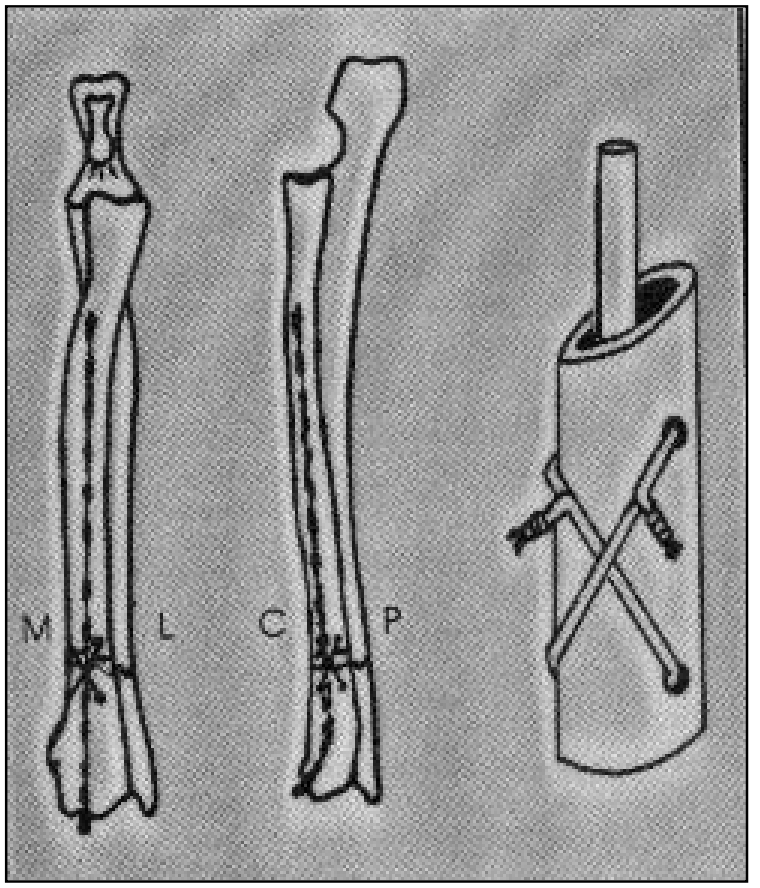

Figura 1 - Forma de colocação no rádio do pino intramedular e dupla hemicerclagem interfragmentária em $X$, na estabilização interna das fraturas distais de rádio e ulna (G I)

palmar, com broca de aço rápido e parafusadeira elétrica, fazendo resfriamento com fluxo contínuo de soro fisiológico. Os pinos utilizados (Steinmann ou Kirschner) tinham pontas trifacetadas em ambas as extremidades, confeccionadas previamente à cirurgia, com um disco de corte específico para aço. A introdução do pino no fragmento distal do rádio foi feita de forma retrógrada, em inclinação tal que saísse o mais cranialmente possível na superfície articular. Efetuada a redução dos fragmentos, o pino foi recalcado no interior do canal medular do fragmento proximal com martelo ortopédico e impactador de pinos. A ponta distal sobressalente foi retirada com cortador de pinos, tentando-se fixar a sobressalente no tecido osteoarticular, mediante impactação final. Em seguida, efetuou-se a fixação dos fios de cerclagem, torcendo-se as extremidades opostas de forma cruzada e em sentido horário, até que houvesse rigidez e imobilidade dos fios. Imediatamente após, cortou-se a porção torcida dos fios, a $1 \mathrm{~cm}$ de sua base, envergando suas extremidades remanescentes no mesmo sentido da torção, para que as extremidades ficassem voltadas para o osso. Após a irrigação dos tecidos com soro físiológico, realizouse a oclusão da ferida cirúrgica em três planos: fáscia muscular, tecido subcutâneo e pele, utilizando-se fio de náilon cirúrgico agulhado. As duas primeiras camadas foram aproximadas com sutura contínua e a

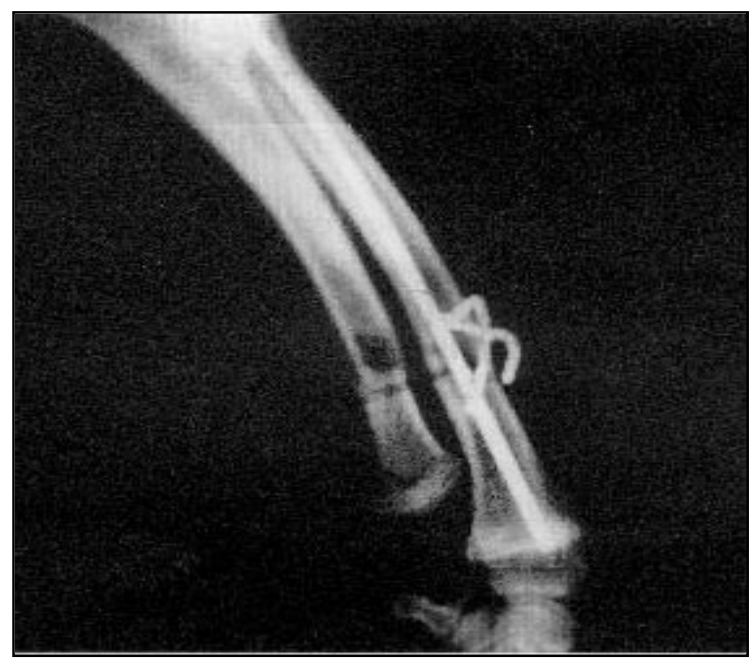

Figura 2 - Radiografia ilustrando a forma de colocação no rádio do pino intramedular e dupla hemicerclagem interfragmentária em X, estabilização interna das fraturas distais de rádio e ilna (Grupo I).

última em pontos isolados simples. Depois da cirurgia, os animais foram medicados com antibióticos (enrofloxacina $-5 \mathrm{mg} / \mathrm{kg}$ e/ou cefalexina $-25 \mathrm{mg} / \mathrm{kg}$, ambos intravenoso) por, no mínimo, sete dias, com antiinflamatório (flunixim meglumine $-1,0 \mathrm{mg} / \mathrm{kg}$, intramuscular) por três dias. Foi feita a imobilização externa do membro operado com algodão ortopédico, tala de PVC, ataduras de crepe e esparadrapo, sendo os animais entregues aos respectivos proprietários.

Após 48 horas de operados, os animais retornaram ao hospital para avaliação da ferida cirúrgica e aferição da temperatura corporal. Passados oito dias, retiraram-se os pontos e refez-se a imobilização externa do membro operado. Aos 30, 60 e 90 dias, fez-se a avaliação radiológica da fratura e, dependendo do resultado, retirou-se a tala de PVC. A restrição de atividade foi mantida até completa consolidação óssea. Quando necessário, observações clínicas posteriores foram realizadas nas residências dos animais.

Nos cães do grupo II, a abordagem craniolateral à diáfise dos ossos rádio e ulna foi efetuada segundo recomendam PIERMATTEI \& GREELEY (1988). O comprimento dos pinos a serem introduzidos variou com o tamanho de cada osso, sendo determinado com o auxílio de uma régua. $\mathrm{O}$ diâmetro dos pinos foi estabelecido por meio de paquímetro de acordo com o canal medular do rádio e da ulna. Os pinos de Steinmann e Kirschner, com pontas em ambas extremidades, foram introduzidos em ambos os ossos de forma retrógrada, até que ficassem expostos $1 / 2 \mathrm{~cm}$ dos pinos nas extremidades fraturadas proximal e distal da ulna e rádio respectivamente. 
Tabela 2 - Resenha, tipo de fratura e evolução dos cães e gatos nos quais se utilizou a técnica de um pino intramedular no rádio e outro na ulna, mais tala, para estabilização de fraturas de rádio e ulna. Recife, 1999.

\begin{tabular}{|c|c|c|}
\hline Resenha & Fratura diafisária & Evolução \\
\hline $\begin{array}{c}11 \text { - Siamês, gato, fêmea, } \\
4 \mathrm{~A}, 3,4 \mathrm{~kg}\end{array}$ & F1/3 PRU ${ }^{1}$ & $\begin{array}{l}\text { Cicatrização óssea normal, } \\
\text { sem retirada dos implantes }\end{array}$ \\
\hline $\begin{array}{c}12 \text { - Siamês, gato, fêmea, } \\
1,2 \mathrm{~A}, 4 \mathrm{~kg}\end{array}$ & $\mathrm{~F} 1 / 3 \mathrm{MRU}^{2}$ & $\begin{array}{l}\text { Cicatrização óssea normal, } \\
\text { sem retirada dos implantes }\end{array}$ \\
\hline $\begin{array}{c}13 \text { - SRD, cão, fêmea, } \\
2 \mathrm{~A}, 8 \mathrm{~kg}\end{array}$ & F1/3 MRU & $\begin{array}{l}\text { Cicatrização óssea normal, } \\
\text { sem retirada dos implantes }\end{array}$ \\
\hline $\begin{array}{c}14 \text { - SRD, cão, macho, } \\
2 \mathrm{~A}, 10 \mathrm{~kg}\end{array}$ & F1/3 MRU & $\begin{array}{l}\text { Cicatrização óssea normal, } \\
\text { sem retirada dos implantes }\end{array}$ \\
\hline $\begin{array}{c}15 \text { - Fila, cão, macho, } \\
3 \mathrm{~A}, 31 \mathrm{~kg}\end{array}$ & F1/3 MRU & $\begin{array}{l}\text { Cicatrização óssea normal, } \\
\text { sem retirada dos implantes }\end{array}$ \\
\hline $\begin{array}{c}16 \text { - SRD, cão, macho, } \\
1,5 \mathrm{~A}, 28 \mathrm{~kg}\end{array}$ & F1/3 MRU & $\begin{array}{l}\text { Cicatrização óssea normal, } \\
\text { sem retirada dos implantes }\end{array}$ \\
\hline $\begin{array}{c}17 \text { - Poodle, cão, macho, } \\
2 \mathrm{~A}, 16 \mathrm{~kg}\end{array}$ & $\mathrm{~F} 1 / 3 \mathrm{MDRU}^{3}$ & $\begin{array}{l}\text { Cicatrização óssea normal, } \\
\text { sem retirada dos implantes }\end{array}$ \\
\hline
\end{tabular}

$\overline{{ }^{1} \text { F1/3 PRU: fratura 1/3 proximal de rádio e ulna; }{ }^{2} \text { F1/3 MRU: fratura } 1 / 3 \text { médio de }}$ rádio e ulna e ${ }^{3}$ F1/3MDRU: Fratura $1 / 3$ médio distal de rádio e ulna.

Após efetuar-se a redução simultânea dos fragmentos de ambos os ossos, mediante a introdução das extremidades dos pinos nos respectivos canais medulares, os pinos foram impactados para o interior

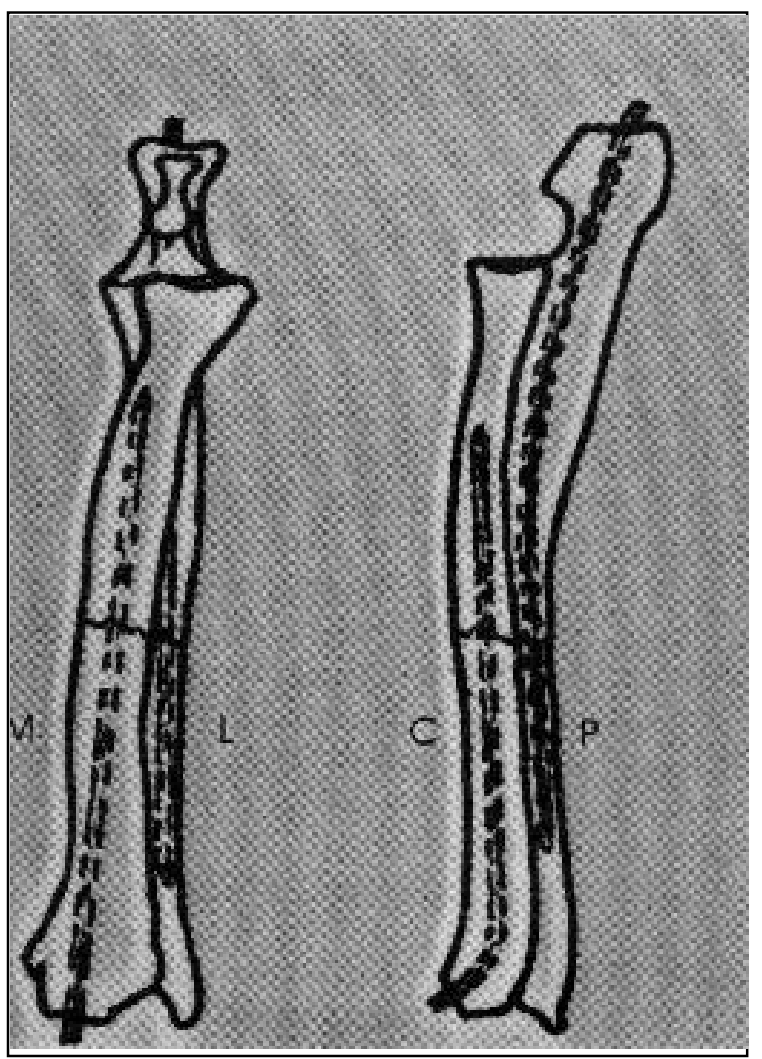

Figura 3 - Forma de colocação dos pinos intramedulares na estabilização das fraturas diafisárias de rádio e ulna (Grupo II). do canal medular do fragmento adjacente, com martelo ortopédico e impactador. Após retirar-se as extremidades excedentes com cortador de pinos, suas pontas remanescentes foram ocultadas conforme descrito no grupo I. A irrigação dos tecidos com soro fisiológico precedeu a oclusão da ferida cirúrgica, que foi suturada em três planos (fascia muscular, tecido subcutâneo e pele), de forma semelhante à realizada no grupo I. Após a cirurgia, os procedimentos referentes à antibioticoterapia, avaliação da ferida cirúrgica, avaliação radiológica e imobilização externa do membro operado foram semelhantes aos efetuados no grupo I.

\section{RESULTADOS}

Em ambos os grupos, todas as feridas cirúrgicas cicatrizaram, sem ocorrência de infecção. A evolução ortopédica dos cães e gatos dos grupos I e II consta nas tabelas 1 e 2 respectivamente. Não união óssea foi observada no animal de $\mathrm{n}^{\mathrm{o}} 2$, pertencente ao grupo I, em que houve a quebra do pino de Kirschner (1mm de espessura) no interior do canal medular. Nos 16 animais restantes, a cicatrização óssea ocorreu entre 30 e 90 dias com formação de calo externo não exuberante. Em nenhum caso houve ruptura das duplas hemicerclagens. No grupo I, exceto nos animais de $\mathrm{n}^{-} 6$ e 9 , não foi necessária a retirada do pino intra-radial, após a consolidação óssea. Em nenhum caso do grupo II, foi necessária a extração dos pinos, visto que os animais não apresentaram sinais de desconforto com os mesmos. O animal $\mathrm{n}^{\mathrm{o}} 10$, pertencente ao grupo I, no qual foi evidenciada claudicação do membro operado, devido à significativa presença do pino no interior da articulação carpo radial, sofreu nova cirurgia para completo sepultamento do pino no rádio. Não foram constatados sinais clínicos e radiográficos de doença degenerativa articular em nenhum dos animais. Em ambos os grupos, ao oitavo dia de operados, $80 \%$ dos animais começavam a apoiar o membro e entre 17 a 20 dias iniciavam a deambulação normal.

\section{DISCUSSÃO}

Em ambos os grupos, a utilização de pinos intramedulares, ao invés de placas e parafusos ou pinos percutâneos mais fixadores externos, reduziu significativamente desvantagens citadas na literatura (PIERMATTEI \& FLO, 1998) sobre estes métodos, como altos custos e necessidade de 


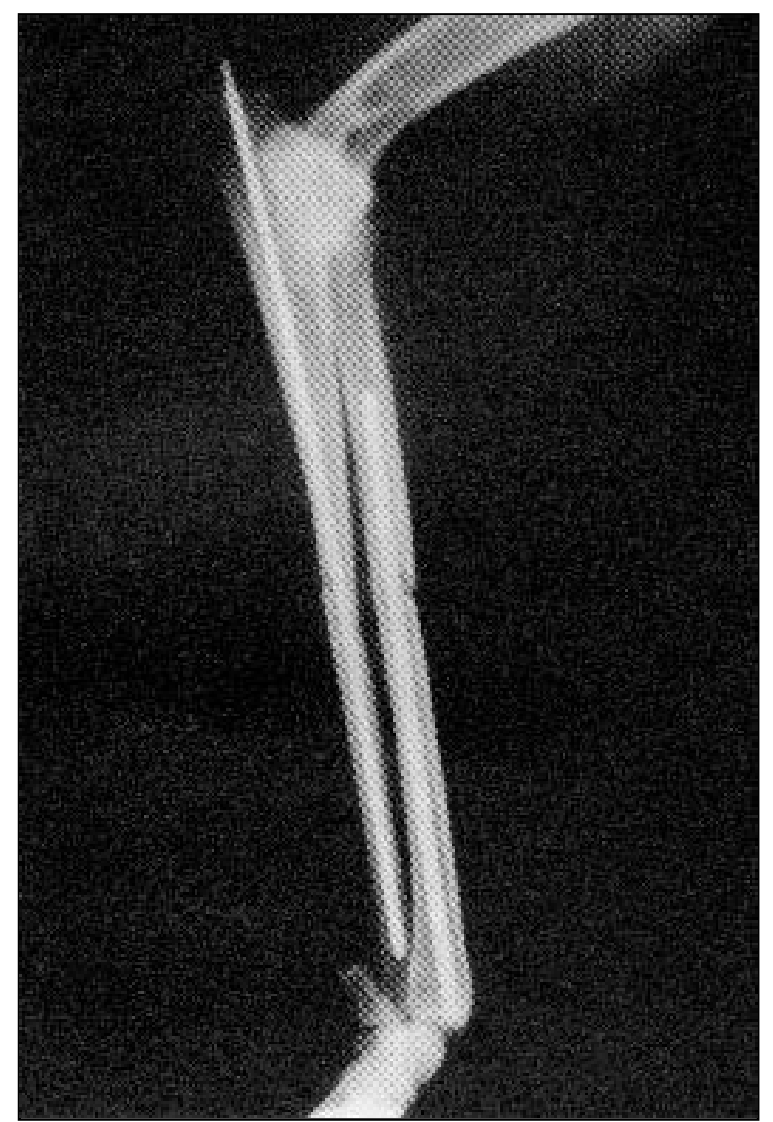

Figura 4 - Radiografia ilustrando a forma de colocação dos pinos intramedulares na estabilização das fraturas diafisárias de rádio e ulna (Grupo II).

instrumental suplementar, cuidados pós-operatórios intensivos, tempo cirúrgico prolongado e extensa exposição óssea.

A complementação com a hemicerclagem em $\mathrm{X}$ ou com o pino ulnar, mais a imobilização externa tiveram influência notória e significativa para reduzir vários dos problemas relacionados ao método de um único pino intramedular no rádio (DEANGELIS et al., 1973; FOSSUM et al., 1997; BOJRAB et $\boldsymbol{a l}$., 1998). Ao invés de incidência de 80\% (LAPPIN et al., 1983), problemas como não união óssea e artropatia observaram-se somente em 2 dos 17 animais $(11,76 \%)$.

A não união óssea rádio-ulnar decorreu da quebra do pino intramedular, o qual, por ser muito fino $(1 \mathrm{~mm})$, não suportou as forças de angulação atuantes no foco de fratura (SINIBALDI \& BOUDREAU, 1992) quando, por descuido do proprietário, o animal retirou a imobilização externa. A impossibilidade de se escolher um pino de diâmetro maior para o rádio, que se adequasse melhor ao peso do animal (FOSSUM et al., 1997; BOJRAB et al., 1998; PIERMATTEI \& FLO, 1998), deveu-se ao reduzido diâmetro ou à forma achatada crânio caudal que normalmente apresenta esse osso (NEWTON \& NUNAMAKER, 1985).

A inclinação implementada durante a introdução retrógrada do pino no fragmento radial distal, somado à tentativa de sepultamento no tecido osteoarticular radial do remanescente do pino, dentro da articulação carporadial, foram essenciais para evitar que ocorressem, na maioria dos animais, claudicação, dor e degeneração articular, complicações citadas por FOSSUM et al., (1997), BOJRAB et al., (1998), PIERMATTEI \& FLO, (1998) referentes à técnica de um pino intramedular radial. A ocorrência em um dos animais foi atribuída a erro de técnica e presença significativa do pino em situação intraarticular, sendo sanada após melhorar o sepultamento da extremidade do mesmo. A discreta saliência intra-articular dos pinos, somado ao crescimento ósseo longitudinal em animais jovens, encobriu as pontas distais dos mesmos, dispensando a necessidade de remoção dos mesmos na maioria dos animais.

A espessura e colocação a $1 \mathrm{~cm}$ da linha de fratura (BLASS et $\boldsymbol{a l}, 1985)$, escolhidas para os fios de cerclagem, demonstraram-se eficientes diannte da ausência de ruptura dos fios e falhas de cicatrização óssea, ocorridas em 9 dos 10 animais do grupo I. A contribuição oferecida pelas cerclagens, conforme citado por SINIBALDI \& BOUDREAU (1992) e BOJRAB et al., (1998), foi de oferecer estabilidade anti-rotacional, a baixo custo. Estas mesmas vantagens foram ofertadas pelo pino na ulna, que ao reforçar a estabilidade angular e impedir deslocamentos rotacionais permitiu rápida recuperação funcional e correta cicatrização óssea, conforme tinha sido citado por SINIBALDI \& BOUDREAU (1992). Enquanto a técnica do pino radial e dupla hemicerclagem interfragmentária em $\mathrm{X}$ parece mais favorável a fraturas rádio-ulnares distais, a de encravamento intramedular em ambos os ossos seria mais adequada para fraturas diafisárias. Justifica-se isso pelo fato de que a ulna é mais acessível junto com o rádio na região das diáfises (PIERMATTEI \& GREELEY, 1988) e também porque o canal medular ulnar fica muito estreito ou inexistente na sua porção distal (NEWTON \& NUNAMAKER, 1985).

\section{CONCLUSÃO}

Com base nos resultados obtidos, recomendam-se as técnicas de um pino intramedular no rádio suplementado com a dupla cerclagem interfragmentária em $\mathrm{X}$ e de um pino intramedular no rádio e ou- 
tro na ulna, ambas complementadas com imobilização externa, para a estabilização de fraturas metafisárias distais e diafisárias, respectivamente, de rádio e ulna, em cães e gatos.

\section{REFERÊNCIAS BIBLIOGRÁFICAS}

ARCHIBALD, J. Canine surgery. 2 ed. Santa Barbara: American Veterinary, 1974. 1172p.

BLASS, C.E, CALDARISE, S.G., TORZILLI P.A., $\boldsymbol{e} \boldsymbol{t}$ al Mechanical properties of three orthopedic wire configurations. American Journal Veterinary Research. v.46, p.1725-1727, 1985 .

BOJRAB, M.J, ELISSON, G.W, SLOCUM, B.C. Currents technics in small animal surgery. 4 ed. Maryland : Williams \& Wilkins, 1998. 340p.

DEANGELIS, M.P, SINIBALDI, K.R, OLDS, R.B., et al. Repair of fractures of the radius and ulna in small dogs. Journal of the American Animal Hospital Association, v.9, p.436-441. 1973.

FOSSUM, T.W, HEDLUND, C.S, HULSE, D.A, et al. Small animal surgery. St. Louis : Mosby, 1997. 1145p.

HERRON, M.R. Repair of distal radio-ulnar fractures in toys breeds. Canine Practice, v.1, p.12-17, 1974.

HINKO, P.J, RHINELANDER, F.W. Effective use of cerclage in the treatment of long-bone fractures in dogs. Journal of the American Veterinary Medical Association, v.166, n.5, p.520-524, 1975 .

KELBER, W.J., CHARLEBOIS G.H. A radically different method for repairing distal radio-ulnar fractures in the dog. Journal of the American Veterinary Medical Association, v.132, p.159, 1958
LAPPIN, M.R, ARON, D.N, HERRON, H.L, et al. Fractures of the radius and ulna in the dog. Journal of the American Animal Hospital Association, v.19, p.643-650, 1983.

MATTHIESEN, D.T. Multiple intramedullary wire fixation of a radial fracture in a dog. Veterinary Surgery, v.13, n.3, p.197-200, 1984.

MUIR, P. Distal antebrachial fractures in toy-breed dogs. Compendium on Continuing Education for the Practicing Veterinarian, v.19, p.137-145, 1997.

NEWTON, D., HOHN, H. Fracture nonunion resulting from cerclage appliance. Journal of the American Veterinary Medical Association, v.164, p.503-508, 1974.

NEWTON, C.D., NUNAMAKER, D.M. Textbook of small animal ortopaedics. Philadelphia : Lippincot, 1985. 1140p.

PIERMATtEI, D.L, GREELEY, R.G. Atlas de abordagens cirúrgicas aos ossos do cão e do gato. 2 ed. São Paulo : Manole, 1988. 195p.

PIERMATTEI, D.L, WIND, A. Orthopedic problems of the lower limbs. Journal of the American Veterinary Medical Association, v.43, p.341, 1976.

PIERMATTEI,D.L, FLO G.L. Small animal orthopedics and fracture repair. 3 ed. Philadelphia : Saunders, 1998. 742p.

SCHWARZ, P.D. Biomechanics of fractures and fracture fixation. Seminars in Veterinary Medicine and Surgery (Small Animal) v.6, p.03-15,1991.

SINIBALDI, K.R, BOUDREAU, R.J. Principles of long bone fracture management. Seminars in Veterinary Medicine and Surgery (Small Animal). v.7, p.44-62, 1992.

WHITTICK, W.G. Traumatologia y ortopedia canina. Barcelona : Aedos, 1978. v.2, 418p.

Ciência Rural, v. 31, n. 1, 2001. 